\title{
ON NONHOMOGENEOUS BOUNDARY VALUE PROBLEM \\ FOR THE STEADY NAVIER-STOKES SYSTEM \\ IN DOMAIN WITH PARABOLOIDAL AND LAYER TYPE OUTLETS TO INFINITY
}

\author{
KRISTINA KAULAKYTE்
}

\begin{abstract}
The nonhomogeneous boundary value problem for the steady Navier-Stokes system is studied in a domain $\Omega$ with two layer type and one paraboloidal outlets to infinity. The boundary $\partial \Omega$ is multiply connected and consists of the outer boundary $S$ and the inner boundary $\Gamma$. The boundary value $\mathbf{a}$ is assumed to have a compact support. The flux of a over the inner boundary $\Gamma$ is supposed to be sufficiently small. We do not impose any restrictions on fluxes of a over the unbounded components of the outer boundary $S$. The existence of at least one weak solution is proved.
\end{abstract}

\section{Introduction}

In this paper we study the nonhomogeneous boundary value problem for the steady Navier-Stokes equations

$$
\begin{cases}-\nu \Delta \mathbf{u}+(\mathbf{u} \cdot \nabla) \mathbf{u}+\nabla p=0 & \text { in } \Omega \\ \operatorname{div} \mathbf{u}=0 & \text { in } \Omega \\ \mathbf{u}=\mathbf{a} & \text { on } \partial \Omega\end{cases}
$$

2010 Mathematics Subject Classification. 35Q30, 35J65, 76D03, 76D05.

Key words and phrases. Navier-Stokes equations, nonhomogeneous boundary value problem, layer type outlet, nonzero flux.

The research leading to these results has received funding from Lithuanian-Swiss cooperation programme to reduce economic and social disparities within the enlarged European Union under project agreement No. CH-3-SMM-01/01. 
in a domain $\Omega \subset \mathbb{R}^{3}$ having layer type and paraboloidal outlets to infinity. Here the vector-valued function $\mathbf{u}=\mathbf{u}(x)=\left(u_{1}(x), u_{2}(x), u_{3}(x)\right)$ and the scalar function $p=p(x)$ are the unknown velocity field and the pressure of the fluid, while $\mathbf{a}(x)=\left(a_{1}(x), a_{2}(x), a_{3}(x)\right)$ is the given boundary value; $\nu>0$ is the constant coefficient of the viscosity.

In bounded domains $\Omega$ with the multiply connected boundaries $\partial \Omega$, consisting of $N$ disjoint components $\Gamma_{j}$, problem (1.1) was studied first by J. Leray in 1933 (see [24]), and thereafter by many authors (see [1]-[9], [12], [15]-[19], [26], [25], [27]-[29], [44], [45], etc.). In case of a bounded domain $\Omega$ continuity equation $\left(1.1_{2}\right)$ implies the necessary compatibility condition for the solvability of problem

$$
\int_{\partial \Omega} \mathbf{a} \cdot \mathbf{n} d S=\sum_{j=1}^{N} \int_{\Gamma_{j}} \mathbf{a} \cdot \mathbf{n} d S=0,
$$

where $\mathbf{n}$ is a unit vector of the outward (with respect to $\Omega$ ) normal to $\partial \Omega$. However, for a long time the existence of a weak solution $\mathbf{u} \in W^{1,2}(\Omega)$ to problem (1.1) was proved either under the condition of zero fluxes

$$
\mathcal{F}_{j}=\int_{\Gamma_{j}} \mathbf{a} \cdot \mathbf{n} d S=0, \quad j=1, \ldots, N,
$$

(e.g. [24], [19], [20], [45]), or assuming the fluxes $\mathcal{F}_{j}$ to be sufficiently small (e.g. [2], [5], [6], [9], [18]), or under the certain symmetry assumptions on the domain $\Omega$ and the boundary value a (e.g. [1], [7], [8], [26], [34]-[36], [16]), or assuming that the arbitrary large flux $\mathcal{F}$ has the "correct" sign (see [15]). Condition (1.3) requires the fluxes $\mathcal{F}_{j}$ of the boundary value a to be zero separately on each connected component $\Gamma_{j}$ of the boundary $\partial \Omega$, while the compatibility condition (1.2) means only that the total flux is equal to zero. Obviously, condition (1.3) is stronger than (1.2), and (1.3) does not allow the presence of sinks and sources. In [24] J. Leray formulated a question whether problem (1.1) is solvable only under the necessary compatibility condition (1.2). In general case this so called Leray's problem was an open problem for 80 years. Fortunately, recently Leray's problem was solved for a 2-dimensional bounded multiply connected domain (see [17]).

In domains with noncompact boundaries problem (1.1) with the homogeneous boundary conditions was exhaustively studied during the last 35 years (e.g. [10], [13], [21]-[23], [33], [37]-[42]). However, not much is known about the nonhomogeneous boundary value problem (1.1) in the domain with noncompact boundaries. To the best of our knowledge problem (1.1) with nonhomogeneous boundary condition for the first time was solved without prescribing a "smallness condition" in 1999 in [30]. Later H. Fujita and H. Morimoto [25]-[27] studied problem (1.1) in the symmetric two-dimensional multiply connected domains $\Omega$ 